\title{
Effect of load distribution on longitudinal and lateral forces acting on each wheel of a compact electric vehicle
}

\author{
Baarath Kunjunni ${ }^{1,2} \cdot$ Muhammad Aizzat bin Zakaria $^{1,2}$ - Anwar P.P Majeed ${ }^{1}$ - A. Fakhri A. Nasir ${ }^{1}$. \\ Mohamad Heerwan Bin Peeie ${ }^{2}$. Umar Zakir Abdul Hamid ${ }^{3}$
}

Received: 7 September 2019 / Accepted: 8 January 2020 / Published online: 21 January 2020

(c) Springer Nature Switzerland AG 2020

\begin{abstract}
Autonomous vehicle is gaining popularity in the market worldwide. Most autonomous vehicle are based on electric vehicles since they are easy to control. The torque control of electric vehicles is precise and easy since electric motor torque can be manipulated by controlling the motor current. Furthermore, the load of the vehicle affects the motor torque of an electric vehicle. A higher vehicle load requires high motor torque to propel the vehicle. Often in autonomous vehicle, the vehicle parameters and stability measure are set within a limit based on standard vehicle settings. However, a vehicle loaded with extra mass on either side can offset these parameters affecting the efficiency of the controller. Thus, in this paper the effect of load on vehicle longitudinal and lateral forces is identified. A simulation model of a two rear in-wheel motored electric vehicle is developed. The model is used to analyze the effect of load on vehicle longitudinal and lateral forces. Based on the result, increasing the load on the side of direction of lateral motion increases the lateral force generated. The high lateral force causes the tires to approach the tire friction circle limit. This can affect the automated vehicle performance since the tires are in unstable region.
\end{abstract}

Keywords Tire forces $\cdot$ Vehicle stability $\cdot$ Nonlinear vehicle model

\section{List of symbols}

$m \quad$ Total mass of the vehicle

$h_{\mathrm{cg}} \quad$ Height of the center of gravity of the vehicle from the ground

$\ddot{x} \quad$ Longitudinal acceleration

$C_{\alpha f} \quad$ Front tire cornering stiffness

$C_{\alpha r} \quad$ Rear tire cornering stiffness

$C_{\sigma} \quad$ Longitudinal tire stiffness

$F_{x i j} \quad$ Longitudinal force with $i=$ front $/$ rear and $j=$ left/ right

$F_{\text {yij }} \quad$ Lateral force with $i=$ front $/$ rear and $j=$ left/right

$I_{z} \quad$ Yaw moment of inertia

$N_{z i j} \quad$ Vertical force acting on each tire with $i=$ front/ rear and $j=$ right/left
$I_{f} \quad$ Length of the front wheels from the center of gravity

load $_{i j} \quad$ Additional load at each wheel with $i=$ front $/$ rear and $j=$ right/left

$I_{r} \quad$ Length of rear wheel from the center of the gravity

$I_{\mathrm{w}} \quad$ Track width

$m_{\text {sij }} \quad$ Static mass at each wheel with $i=$ front $/$ rear and $j=$ left/right

$\ddot{y} \quad$ Lateral acceleration

$\ddot{\varphi} \quad$ Yaw acceleration

$K \quad$ Road constant

$g$ Gravitational acceleration

I Sum of $I_{\mathrm{f}}$ and $I_{\mathrm{r}}$

$\alpha \quad$ Tire slip angle

$\triangle$ Muhammad Aizzat bin Zakaria, maizzat@ump.edu.my $\mid{ }^{1}$ iMAMs Laboratory, Faculty Manufacturing and Mechatronic Engineering Technology, Universiti Malaysia Pahang, 26600 Pekan, Pahang, Malaysia. ${ }^{2}$ Autonomous Vehicle Laboratory, Automotive Engineering Technology (AEC), Universiti Malaysia Pahang, 26600 Pekan, Pahang, Malaysia. ${ }^{3}$ Sensible 4, Otakaari 5 I / 5 A, 02150 Espoo, Finland. 
$\delta \quad$ Wheel steering angle

$\mu \quad$ Tire-road friction coefficient

$\rho \quad$ Tire slip ratio

\section{Introduction}

Development of autonomous vehicle depends on the information on the vehicle parameters and forces acting on it. A working autonomous vehicle system requires information on various vehicle parameters to drive the vehicle safely. The expansion of the autonomous vehicle industry is influenced by the development of electric vehicles due to its capability to accommodate precise control strategy $[1,2]$. An autonomous vehicle control is designed with active elements to ensure the stability of the vehicle [3]. Few of such active safety elements are anti-lock braking system (ABS), adaptive cruise control (ACC), dynamic stability program (DSC) and electronic stability program (ESP) [4-6].

Most of the active safety controls are developed by using vehicle dynamic model which requires the vehicle lateral and longitudinal forces as an input in determining the vehicle states such as longitudinal acceleration, lateral acceleration, yaw rate and body slip angle. Most of the aforementioned vehicle states can be measured using instrumental sensors such as GPS and accelerometer [3]. However, such sensors are also unreliable due to their limitations such as loss of signals, effect of weather and high cost. Thus, Youssfi et al., 2018 suggested estimation of vehicle parameters using vehicle dynamic models. Moreover, estimation of vehicle parameters is widely used by many researchers in their quest to develop a robust controller [4] or estimating vehicle parameters and stability [7]. A vehicle dynamic model of the respective vehicle is required to implement the vehicle parameter estimation model.

An insight on the vehicle dynamic models highlights the importance of lateral and longitudinal forces acting on the wheels. These two forces are prominent in determining the behavior of the car based on the vehicle dynamic models such as linear and nonlinear models. The linear model highlights the longitudinal and lateral vehicle motion independently. On the other hand, the nonlinear model shows the dependence of the longitudinal and lateral motion. Furthermore, the development of vehicle dynamic model requires the inclusion of the tire model since the tires play an important role in determining the vehicle characteristic. However, there are few different tire models available to describe the tire characteristic. The tire model is used to determine the lateral and longitudinal forces acting on each wheel. Tire models such as magic formula [8], LuGre [9] and Dugoff's tire model [2] are few of the commonly used models. Furthermore, most of the tire models are dependent on the vehicle vertical force acting $\left(N_{z}\right)$ on each tire. The vertical force is often calculated using the load transfer equation [10]. The load transfer equation requires the mass of the vehicle to estimate the vertical force. However, increment of mass on either side of the vehicle can affect the vehicle stability. Often, only vehicle mass is considered in the study by researchers; however, this is not true since passengers or goods are loaded into the vehicle. The distribution of the additional load on the vehicle can affect the forces acting on each wheel, which in turn affect the vehicle parameters and stability.

This paper focuses on identifying the effect of load distribution for two in-wheel motored electric vehicles toward vehicle lateral and longitudinal forces.

\section{Model development}

The two in-wheel motored electric vehicle is modeled by integrating the DC motor model, vehicle dynamic nonlinear model, load transfer model and the Dugoff's tire model. The vehicle model is designed based on rear wheel motored electric vehicle. In this research, Dugoff's tire model is implemented since it is a pure empirical model which does not require parameters determined experimentally.

\subsection{Vehicle dynamic model}

The vehicle motion is determined through the vehicle dynamic model which comprises of longitudinal and lateral motion. The vehicle dynamic motion equation is determined based on Fig. 1.

The vehicle longitudinal motion is represented by Eq. (1), whereas the lateral motion is represented by Eq. (2). On the other hand, Eq. (3) is used to calculate the vehicle yaw rate.

$m \ddot{x}=\left(F_{x f l}+F_{x f r}\right) \cos (\delta)+F_{x r l}+F_{x r r}-\left(F_{y f l}+F_{y f r}\right) \sin (\delta)+m \dot{\varphi} \dot{y}$

$m \ddot{y}=F_{y r l}+F_{y r r}+\left(F_{x f l}+F_{x f r}\right) \sin (\delta)+\left(F_{y f l}+F_{y f r}\right) \cos (\delta)-m \dot{\varphi} \dot{x}$ 
Fig. 1 Vehicle dynamic model representation

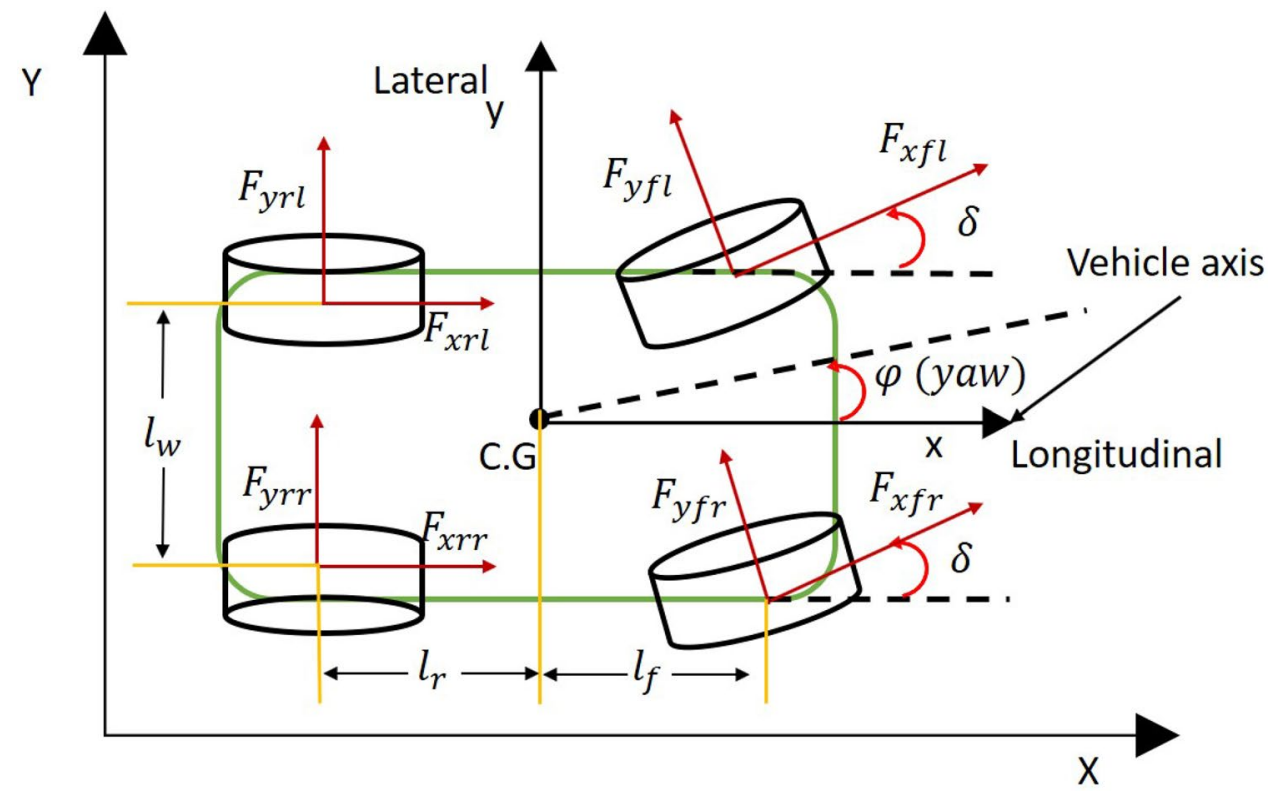

$I_{z} \ddot{\varphi}=I_{\mathrm{f}}\left(F_{x f l}+F_{x f r}\right) \sin (\delta)+I_{\mathrm{f}}\left(F_{y f l}+F_{y f r}\right) \cos (\delta)-I_{\mathrm{r}}\left(F_{y r l}+F_{y r r}\right)$

$+\frac{I_{\mathrm{w}}}{2}\left(F_{x f r}-F_{x f l}\right) \cos (\delta)+\frac{I_{\mathrm{w}}}{2}\left(F_{x r r}-F_{x r l}\right)+\frac{I_{\mathrm{w}}}{2}\left(F_{y f l}-F_{y f r}\right) \sin (\delta)$

Based on Eqs. (1) to (3), it is evident that the lateral and longitudinal forces play an important role in the vehicle motion estimation.

\subsection{Dugoff's tire model}

In order to determine the vehicle lateral and longitudinal forces, the Dugoff's tire model is adopted due to its simple nature and relationship between tire forces, tire-road friction and vertical force.

$\tau=\frac{\mu N_{z i j}(1+\rho)}{2\left[\left(C_{\sigma} \rho\right)^{2}+\left(C_{\alpha} \tan (\alpha)\right)^{2}\right]^{1 / 2}}$

$f(\tau)=(2-\tau) \tau$ if $\tau<1$

$f(\tau)=1$ if $\tau>1$

The longitudinal and lateral forces are calculated using Eq. (7) and (8).

$$
\begin{aligned}
& F_{x i j}=C_{\sigma} \frac{\rho}{1+\rho} f(\tau) \\
& F_{y i j}=C_{\alpha} \frac{\tan \alpha}{1+\rho} f(\tau)
\end{aligned}
$$

\subsection{Load transfer model}

Both longitudinal and lateral tire forces depend on the vertical force acting on each tire. The vehicle vertical force is determined from the load transfer model which is adapted from [11]. The load transfer model considers both longitudinal and lateral load transfer which requires longitudinal and lateral acceleration of the vehicle, as shown in Eqs. (9) to (12). The load transfer model is divided into three parts: static force, longitudinal load transfer and lateral load transfer. The additional load introduced on the vehicle is added to the static force of the vehicle. In this study, the initial mass on each tire is considered equal. However, a set of loads is added on each tire to simulate an instance of unbalanced overloading.

$N_{z f r}=\left(m_{s i j}+\operatorname{load}_{i j}\right) g \frac{l_{\mathrm{r}}}{l}+\frac{m a_{y} h_{\mathrm{cg}}}{l_{\mathrm{w}}}-m a_{x} \frac{h_{\mathrm{cg}}}{l}$

$N_{z f l}=\left(m_{s i j}+\operatorname{load}_{i j}\right) g \frac{l_{\mathrm{r}}}{l}-\frac{m a_{y} h_{\mathrm{cg}}}{l_{\mathrm{w}}}-m a_{x} \frac{h_{\mathrm{cg}}}{l}$

$N_{z r r}=\left(m_{s i j}+\operatorname{load}_{i j}\right) g \frac{l_{\mathrm{f}}}{l}+\frac{m a_{y} h_{\mathrm{cg}}}{l_{\mathrm{w}}}+m a_{x} \frac{h_{\mathrm{cg}}}{l}$ 


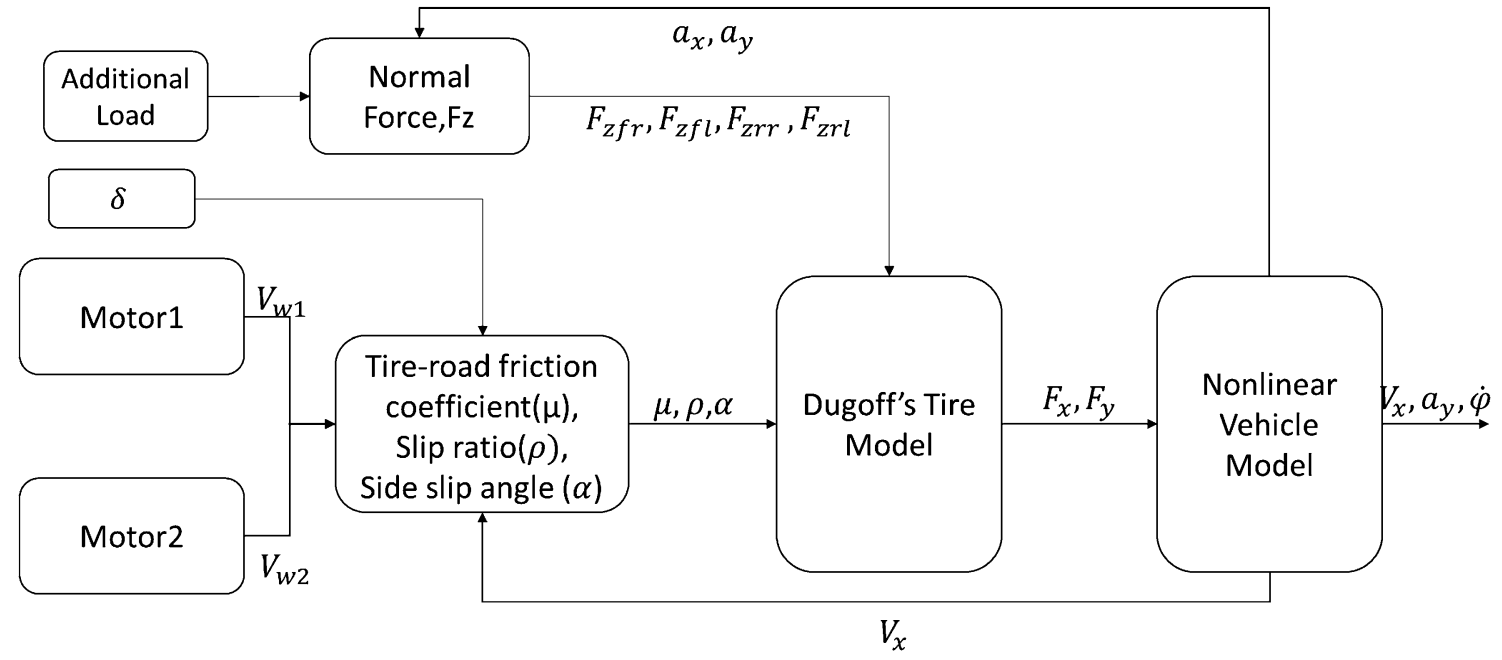

Fig. 2 Simulation model representation

Fig. 3 Wheel steering input
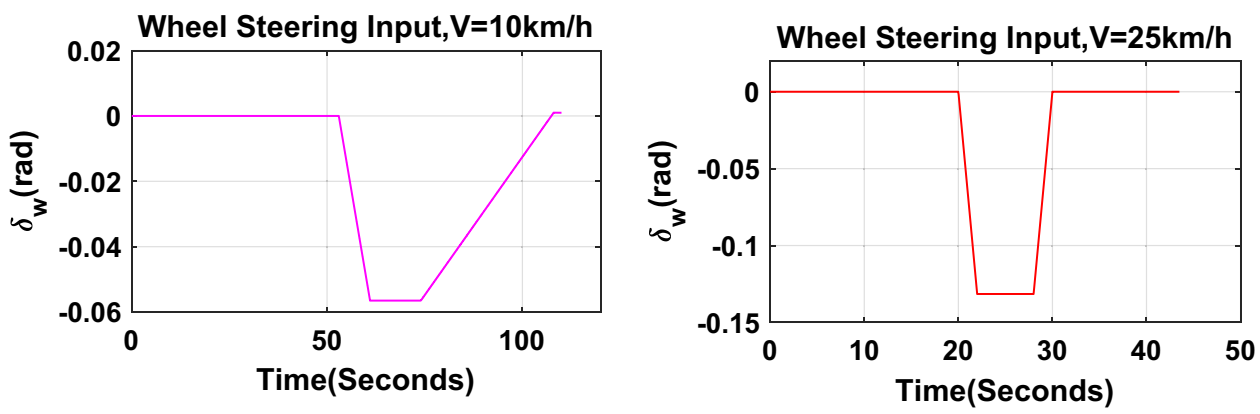

$N_{z r l}=\left(m_{s i j}+\operatorname{load}_{i j}\right) g \frac{I_{\mathrm{f}}}{l}-\frac{m a_{y} h_{\mathrm{cg}}}{I_{\mathrm{w}}}+m a_{x} \frac{h_{\mathrm{cg}}}{l}$

The inertial acceleration of the vehicle along the $y$ axis is given by $a_{y}$ and is measured using Eq. (13). On the other hand, the inertial acceleration of the vehicle along the $x$-axis is given by $a_{x}$ and is measured using Eq. (14).

$a_{y}=\ddot{y}+V_{x} \dot{\varphi}$

$a_{x}=\ddot{x}-V_{y} \dot{\varphi}$

\subsection{Simulation setup}

The simulation is designed using MATLAB Simulink. The simulation model representation is as shown in Fig. 2. The simulation model includes the DC motor, Dugoff's tire model, vehicle dynamic model and load transfer model. Furthermore, the model is tested at two different velocities of $10 \mathrm{~km} / \mathrm{h}$ (low) and $25 \mathrm{~km} / \mathrm{h}$ (high) since the vehicle used is a compact electric vehicle (EV) with max velocity of $30 \mathrm{~km} / \mathrm{h}$. The EV is given a steering input to turn around
Table 1 Percentage of load distribution on the right

\begin{tabular}{llll}
\hline $\begin{array}{l}\text { Percent- } \\
\text { age }(\%)\end{array}$ & Total load (kg) & Front load $(\mathrm{kg})$ & Rear load $(\mathrm{kg})$ \\
\hline 5 & 12.97 & 6.48 & 6.48 \\
10 & 25.93 & 12.97 & 12.97 \\
15 & 38.90 & 19.45 & 19.45 \\
20 & 51.86 & 25.93 & 25.93 \\
25 & 64.83 & 32.41 & 32.41 \\
30 & 77.80 & 38.89 & 38.89 \\
35 & 90.76 & 45.38 & 45.38 \\
40 & 103.72 & 51.86 & 51.86 \\
\hline
\end{tabular}

a constant radius as shown in Fig. 3 Furthermore, Table 1 shows the value of additional load distributed at the right side of the vehicle. Only the effect of additional load on the right side is discussed to highlight the extent of variation in force that occurs during a right cornering motion. The additional load is measured as the percentage of the 
Table 2 Vehicle parameter and physical measurement

\begin{tabular}{lll}
\hline Symbol & Value & Units \\
\hline$I_{\mathrm{f}}$ & 0.53 & $\mathrm{~m}$ \\
$I_{\mathrm{r}}$ & 0.62 & $\mathrm{~m}$ \\
$C_{\alpha f}$ & 2000 & $\mathrm{~N} / \mathrm{rad}$ \\
$C_{\alpha r}$ & 2612.62 & $\mathrm{~N} / \mathrm{rad}$ \\
$C_{\sigma}$ & 3000 & $\mathrm{~N} / \mathrm{m}^{3}$ \\
$k$ & $1=$ dry asphalt & - \\
$g$ & 9.81 & $\mathrm{~m} / \mathrm{s}^{2}$ \\
$m$ & 322.1 & $\mathrm{~kg}$ \\
$h_{\mathrm{cg}}$ & 0.105 & $\mathrm{~m}$ \\
$I_{\mathrm{w}}$ & 1.43 & $\mathrm{~m}$ \\
$I_{z}$ & 1470 & $\mathrm{kgm}$ \\
\hline
\end{tabular}

Table 3 Static mass of each tire

\begin{tabular}{llll}
\hline$M_{f r}$ & $M_{f l}$ & $M_{r r}$ & $M_{r l}$ \\
\hline \multicolumn{3}{l}{ Mass } & with driver \\
88 & 71 & 70.5 & 92.6 \\
\hline
\end{tabular}

vehicle mass. In this simulation testing, the mass of the vehicle is measured from a compact electric vehicle.

The vehicle parameter and physical measurement are represented in Table 2 . The vehicle physical parameters including $l_{\mathrm{f}}, l_{\mathrm{r}}, h_{\mathrm{cg}}$ and $m$ are measured from a compact electric vehicle. Other parameters are adopted from the following paper [12].

The static mass of the vehicle is measured by placing a weighing scale below each tire, and the corresponding mass is listed in Table 3. Furthermore, the static mass measured is inclusive of the mass of the driver.

The wheel steering input for the vehicle at $10 \mathrm{~km} / \mathrm{h}$ and $20 \mathrm{~km} / \mathrm{h}$ is given at two different times in order to facilitate the same turn radius as depicted in Fig. 4.

\section{Results and discussion}

The vehicle is simulated at two velocities: 10 and $25 \mathrm{~km} / \mathrm{h}$. For each vehicle velocity, the vehicle is added with load at the right side in an increment of $5 \%$. The vehicle tire forces are observed and displayed in Figs. 5 , 6,7 and 8 . The vehicle tire forces with respect to the planar motion are divided into longitudinal and lateral forces. For the first part, the vehicle longitudinal force is studied as shown in Figs. 5 and 6. Based on Fig. 5, the longitudinal force on each tire varies as the load is increased on the right side. As observed, the right tire experiences more longitudinal force compared to the left tire. Furthermore, the right tire experiences highest longitudinal force at $40 \%$ increased load compared to the no-load condition ( $0 \%)$.

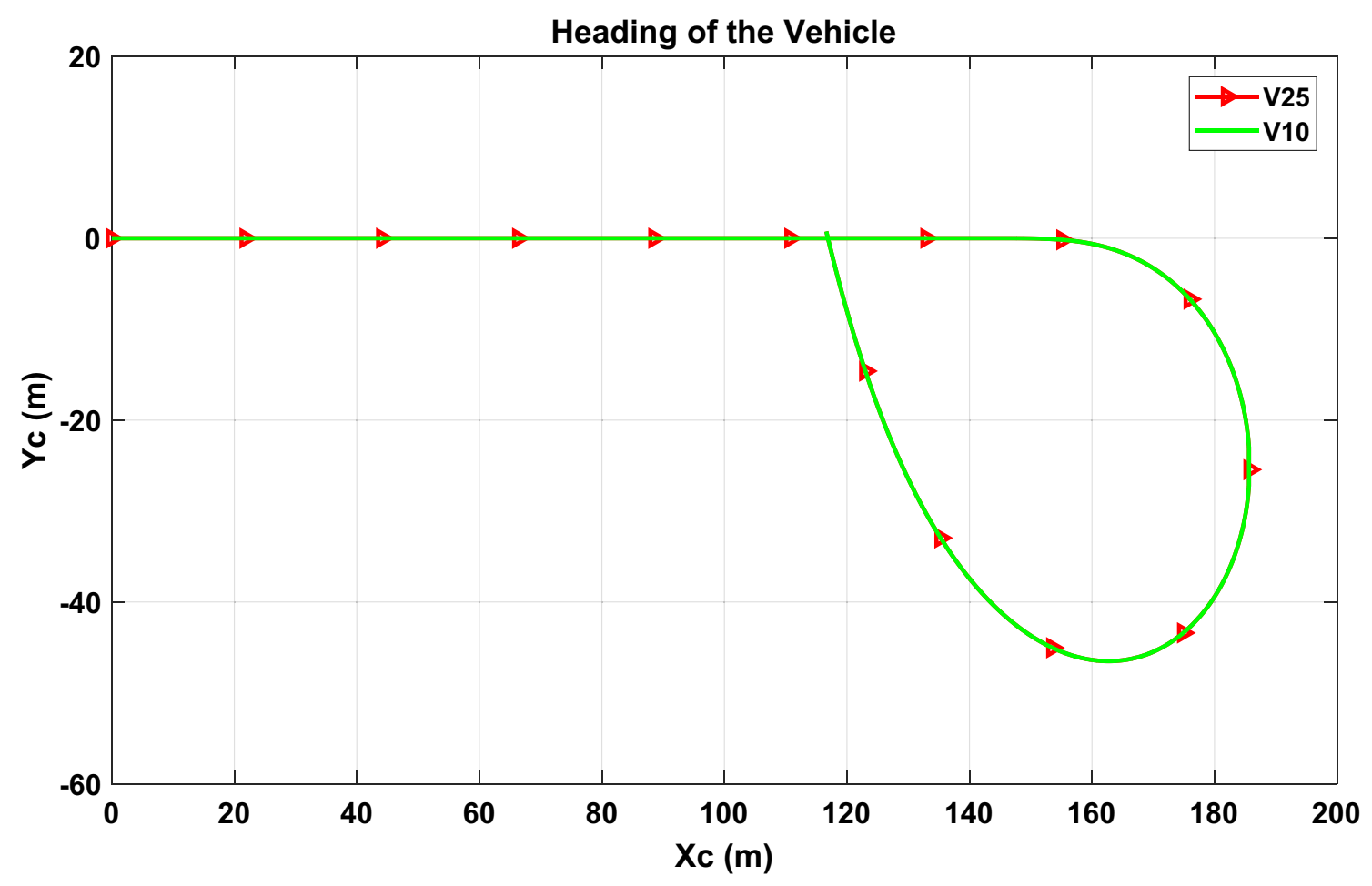

Fig. 4 Direction of motion of the vehicle at $10 \mathrm{~km} / \mathrm{h}$ and $25 \mathrm{~km} / \mathrm{h}$ 

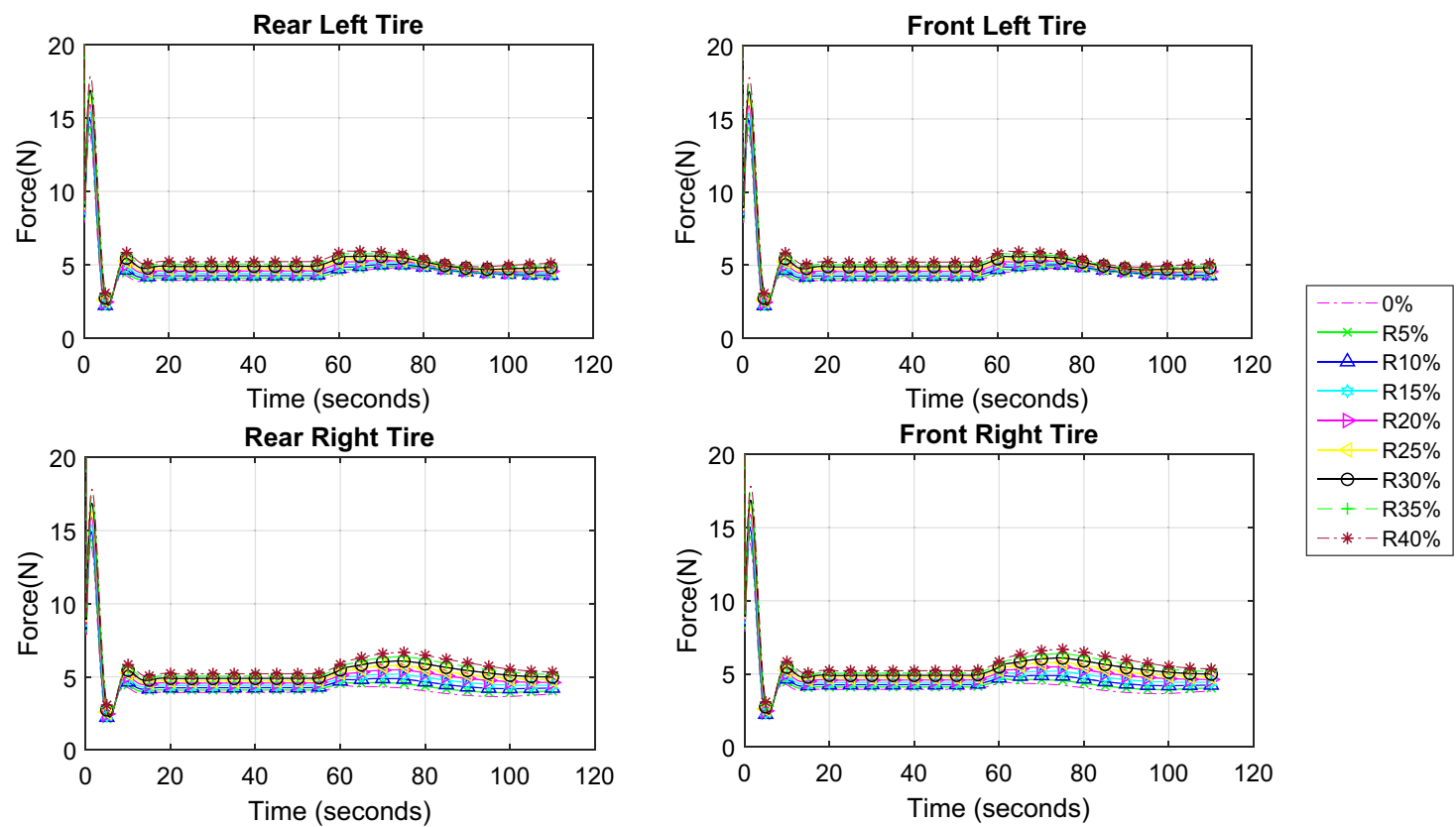

Fig. 5 Longitudinal force at each tire for $V=10 \mathrm{~km} / \mathrm{h}$
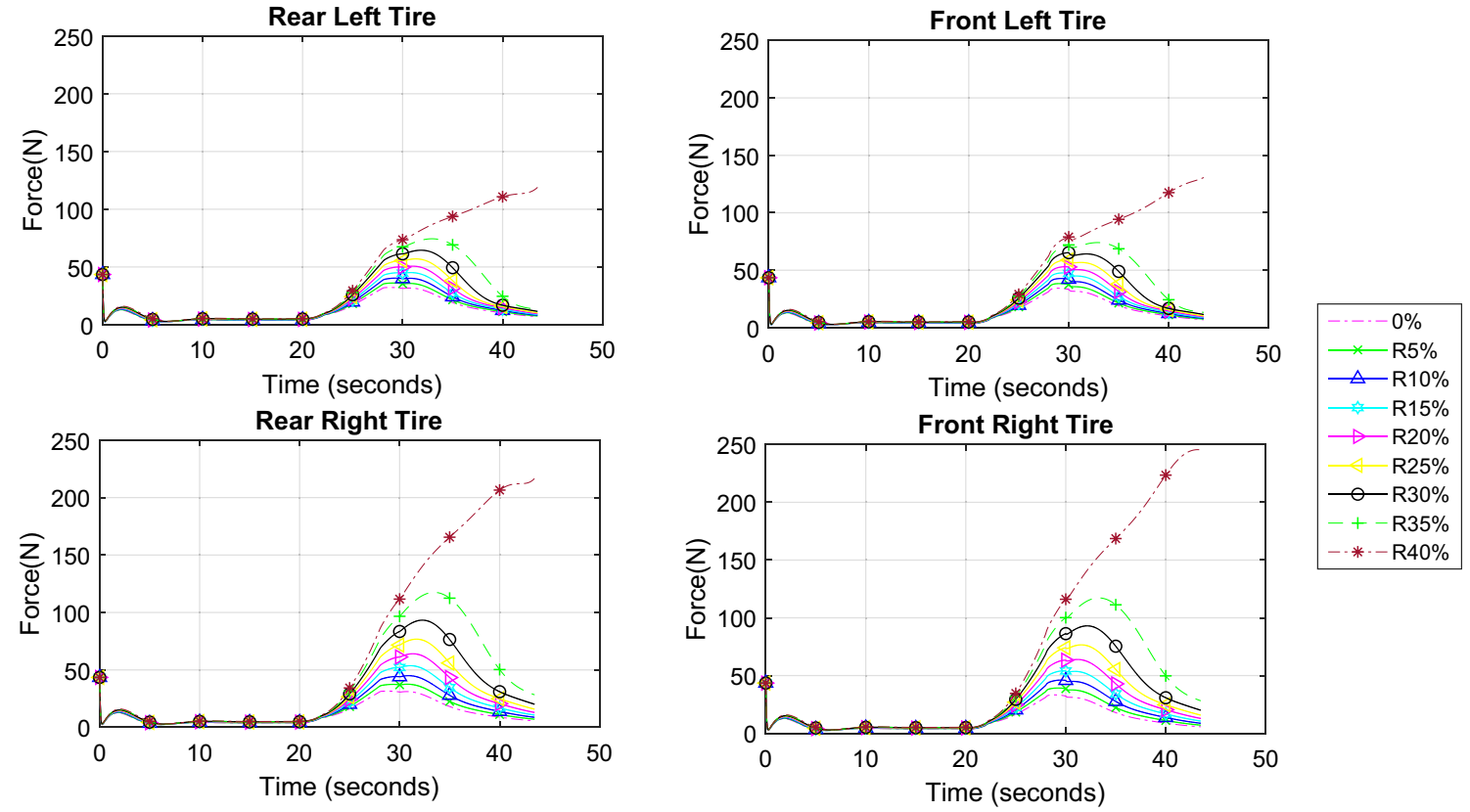

Fig. 6 Longitudinal force at each tire for $V=25 \mathrm{~km} / \mathrm{h}$

Similarly, the vehicle traveling at $25 \mathrm{~km} / \mathrm{h}$ experiences increased longitudinal force at each tire with increase in additional right load. However, at higher velocity ( $25 \mathrm{~km} / \mathrm{h})$, the longitudinal force generated at each tire is far greater than the vehicle traveling at $10 \mathrm{~km} / \mathrm{h}$. Based on Fig. 6 , the longitudinal force at each tire increases with increasing load. Furthermore, at $40 \%$ increased load, the tire force increases continuously. This condition occurs when the tire starts slipping.

The lateral force acting on each tire for vehicle traveling at $10 \mathrm{~km} / \mathrm{h}$ and $25 \mathrm{~km} / \mathrm{h}$ is depicted in Figs. 7 and 8 . Based on Fig. 7, the lateral force at each tire increases with the additional load. The lateral force is generated when the vehicle is taking a right corner. Thus, it can be observed 

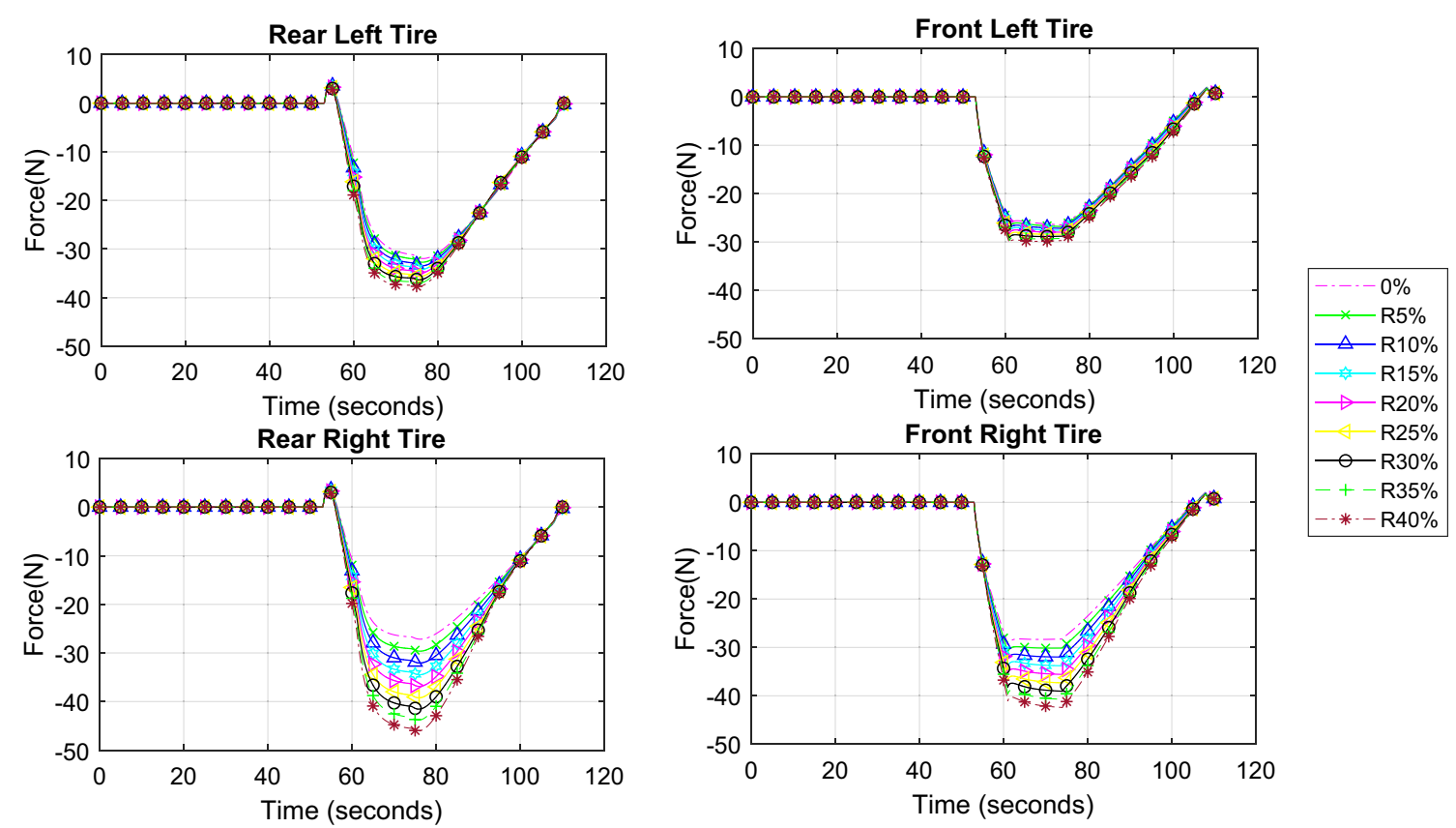

Fig. 7 Lateral force at each tire for $V=10 \mathrm{~km} / \mathrm{h}$
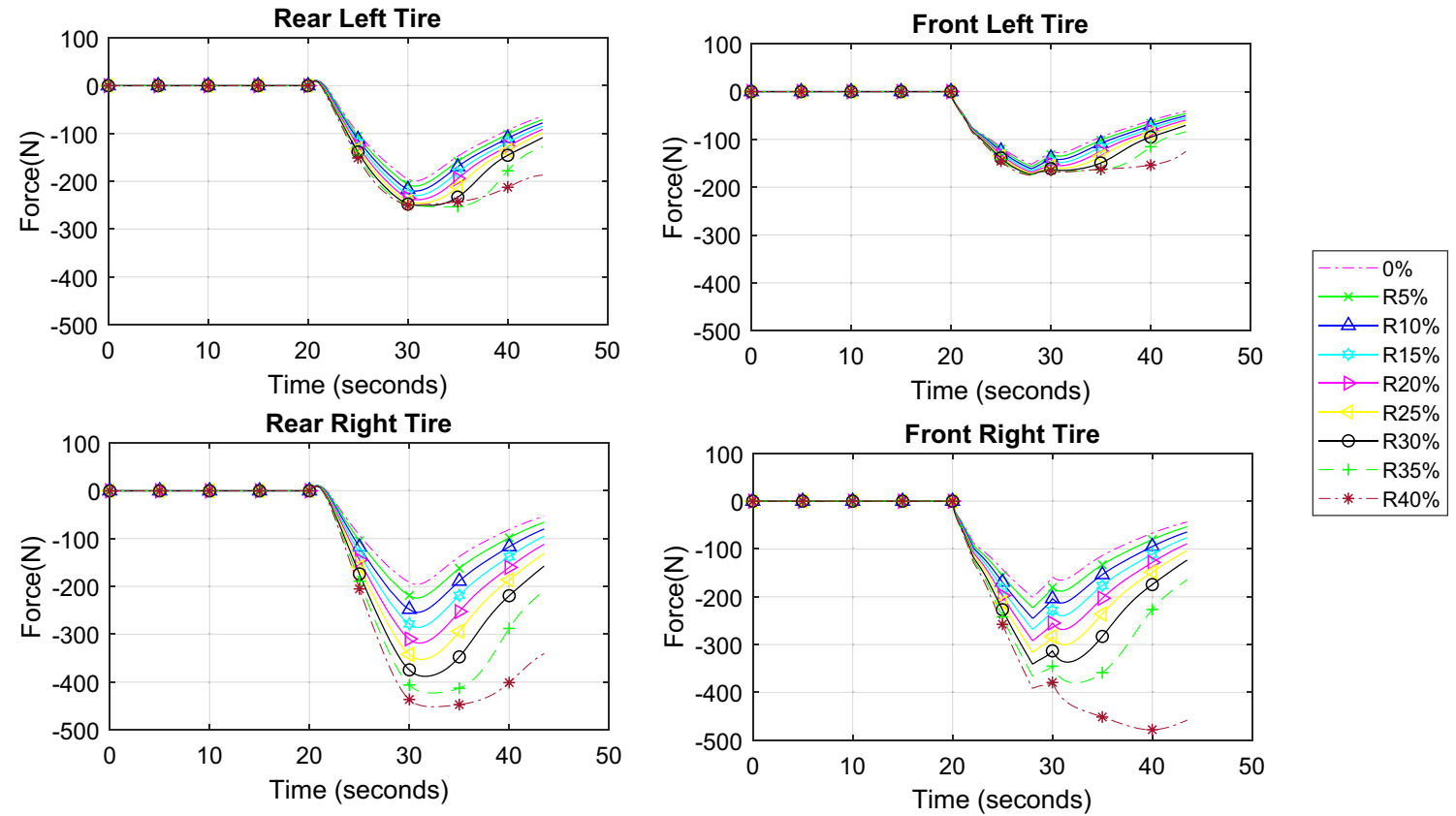

Fig. 8 Lateral force at each tire for $V=25 \mathrm{~km} / \mathrm{h}$

that the tire force at the right is higher than that at the left. This is because the inner tire experiences more force during cornering. Furthermore, the right tires have the highest lateral force when an additional load of $40 \%$ is added. This shows that increasing the additional load at the tires can increase the tire's lateral force.
Similarly, Fig. 8 depicts the lateral force at each tire for a vehicle traveling at $25 \mathrm{~km} / \mathrm{h}$. The lateral force at the right tires increases with increasing load. The front right tire is observed to have a larger effect when an additional load of $40 \%$ is added. The lateral force at the front right tire is almost 500 N. Furthermore, comparing Figs. 7 and 8 shows that the lateral force experienced by the right tires at high 
velocity is approximately 10 times higher than that at low velocity. This shows that at higher velocity, the tires experience higher lateral force during cornering. The lateral force is also affected by the additional load on the tires. Increasing more load on the tires increases the lateral force.

Based on the observation on Figs. 5, 6, 7 and 8, the force acting on each tire increases with the increase in load at respective tires. The correlation between vertical force and longitudinal and lateral force can be observed from Eqs. (4), (7) and (8). Increased load on each tire increases the vertical force acting on the tires toward the road. The increased vertical force causes the vehicle to generate higher longitudinal force to move the vehicle forward. Furthermore, a higher lateral force is generated to facilitate the required motion due to the high vertical force acting on the respective tires. Moreover, the longitudinal and lateral forces acting on the right tire increase drastically at high velocity ( $25 \mathrm{~km} / \mathrm{h}$ ). At the highest load (40\%), both right tires experience continuously increasing longitudinal force compared to other load increment setting. This shows that there is a limit for the amount of load that can be sustained by a tire at high velocity before experiencing instability. This scenario is also observable for the lateral force at the front right tire for the vehicle traveling at $25 \mathrm{~km} / \mathrm{h}$.

\section{Conclusion and recommendations}

There is a significant effect of load distribution on the longitudinal and lateral forces acting on each tire. The additional load added on the side of the vehicle lateral motion induces increased longitudinal and lateral tire force. Based on the result, increasing load on the right side of the vehicle above a certain limit causes the vehicle to crash. This can be observed at the vehicle traveling at $25 \mathrm{~km} / \mathrm{h}$ with a right load of $40 \%$. The longitudinal force generated at the tires increases continuously, depicting the tire slip ratio exceeded one. The tire slip ratio for a stable driving condition is limited below one. Therefore, it can be concluded that increasing load on the same side of the vehicle lateral motion can cause the tire longitudinal and lateral forces to increase. Increasing the load above a limit causes the vehicle to experience instability.

For future work, the study can be enhanced by including the effect of load distribution at the opposing side of the lateral motion. For example, the load can be added to the left side of vehicle taking a right turn. Furthermore, the vehicle stability can be analyzed using the stability criteria such as rollover index, friction circle coefficient and lateral acceleration limit.
Acknowledgements The authors would like to thank for the financial support of the project provided under the Fundamental Research Grant Scheme (FRGS) no. RDU190104 by Ministry of Education, Malaysia.

\section{Compliance with ethical standard}

Conflict of interest The authors declare that they have no conflict of interest.

\section{References}

1. Wu D, Li Y, Zhang J, Du C (2018) Torque distribution of a four inwheel motors electric vehicle based on a PMSM system model. Proc Inst Mech Eng Part D J Automob Eng 232(13):1828-1845

2. Jia F, Liu Z, Zhou H, Chen W (2018) A novel design of traction control based on a piecewise-linear parameter-varying technique for electric vehicles with in-wheel motors. IEEE Trans Veh Technol 67(10):9324-9336

3. El Youssfi N, Oudghiri M, Aitouche A, El Bachtiri R (2018) Fuzzy sliding-mode observer for lateral dynamics of vehicles with consideration of roll motion. In: 2018 26th mediterranean conference on control and automation (MED) pp. 861-866

4. Zhang L, Ding H, Guo K, Zhang J, Pan W, Jiang Z (2019) Cooperative chassis control system of electric vehicles for agility and stability improvements. IET Intell Transp Syst 13(1):134-140

5. Wang Y, Wang Z, Zhang L, Liu M, Zhu J (2019) Lateral stability enhancement based on a novel sliding mode prediction control for a four-wheel-independently actuated electric vehicle. IET Intell Transp Syst 13(1):124-133

6. Wang J, Luo Z, Wang Y, Yang B, Assadian F (2018) Coordination control of differential drive assist steering and vehicle stability control for four-wheel-independent-drive EV. IEEE Trans Veh Technol 67(12):11453-11467

7. Zhao YQ, Li HQ, Lin F, Wang J, Ji XW (2017) Estimation of road friction coefficient in different road conditions based on vehicle braking dynamics. Chin J Mech Eng 30(4):982-990

8. Kunnappillil Madhusudhanan A, Corno M, Arat MA, Holweg E (2016) Load sensing bearing based road-tyre friction estimation considering combined tyre slip. Mechatronics 39:136-146

9. Hashemi E, Pirani M, Khajepour A, Kasaiezadeh A (2016) A comprehensive study on the stability analysis of vehicle dynamics with pure/combined-slip tyre models. Veh Syst Dyn 54(12):1736-1761

10. Mashadi B, Mostaghimi H (2017) Vehicle lift-off modelling and a new rollover detection criterion. Veh Syst Dyn 55(5):704-724

11. K. Baarath, M. A. Zakaria, N. A. Zainal (2018) An investigation on the effect of lateral motion on normal forces acting on each tires for nonholonomic vehicle, In: Intelligent manufacturing \& mechatronics, Springer, Singapore, pp 611-621

12. Ishak MI, Ogino H, Yamamoto $Y$ (2016) Numerical simulation analysis of an oversteer in-wheel small electric vehicle integrated with four-wheel drive and independent steering. Int J Veh Technol 2016:1-12

Publisher's Note Springer Nature remains neutral with regard to jurisdictional claims in published maps and institutional affiliations. 